\title{
A unique technique for the removal of threaded biliary inside plastic stents
}

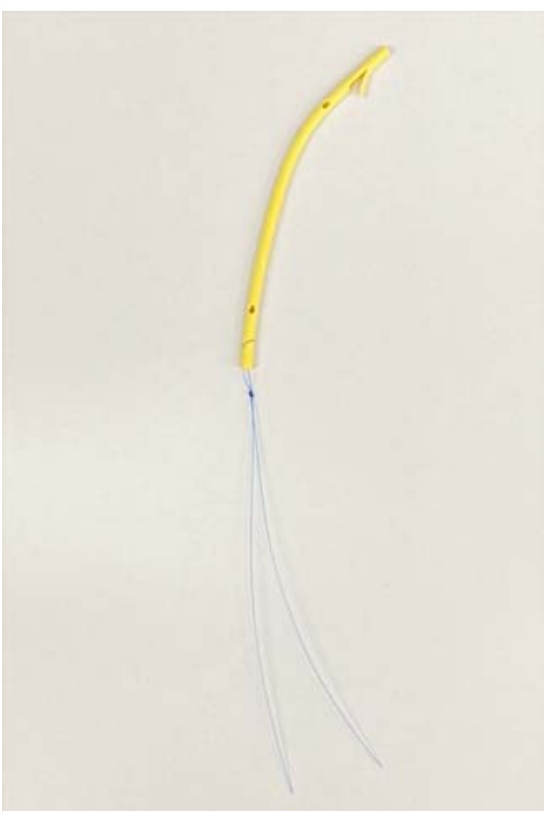

Fig. 1 Image of a threaded inside plastic stent (Through \& Pass; Gadelius Medical, Tokyo, Japan).

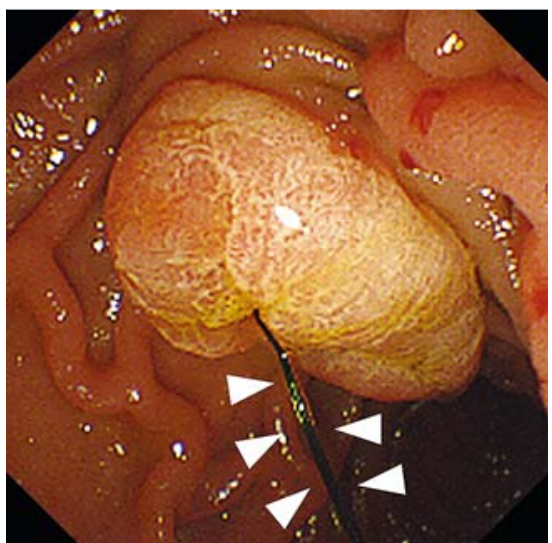

- Fig. 3 Duodenoscopy image showing the thread of the inside stent (white arrowheads).

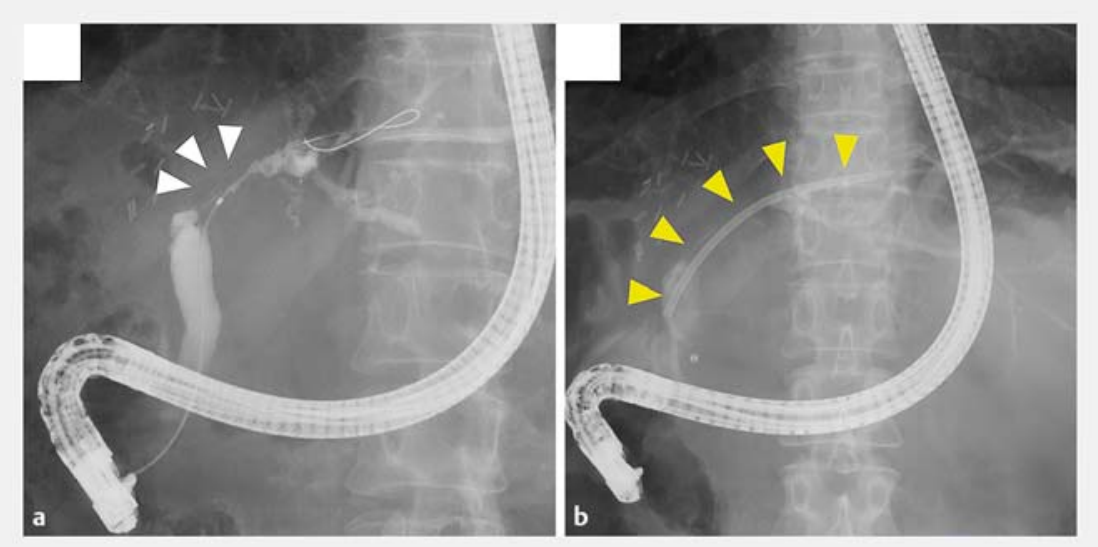

- Fig. 2 Endoscopic retrograde cholangiography images showing: a the refractory biliary stricture after living donor liver transplantation (white arrowheads); $\mathbf{b}$ the inside stent previously placed across the biliary stricture (yellow arrowheads).

A 48-year-old woman underwent living donor liver transplantation (LDLT) for primary sclerosing cholangitis 10 years ago. To manage a refractory anastomotic biliary stricture after LDLT, a plastic stent was placed in the bile duct as an inside stent (above the sphincter of Oddi) and endoscopic retrograde cholangiography (ERCP) was performed periodically for inside stent replacement. Most recently, a nylon-threaded plastic stent (8.5- $\mathrm{Fr} \times$ 9-cm; Through \& Pass; Gadelius Medical, Tokyo, Japan) had been placed as an inside stent across the biliary stricture 6 months previously ( $\bullet$ Fig. 1 and $\triangleright$ Fig. 2 ). Duodenoscopy now identified the thread that was emerging into the duodenum ( $\triangleright$ Fig. 3 ). In our technique, first, the thread was gripped using a rotatable grasping forceps (FG-44NR-1; Olympus, Tokyo, Japan) ( $\mathbf{F i g . 4 ) . ~ S e c o n d , ~ t h e ~}$ grasping forceps was slowly rotated to roll the thread as if twirling spaghetti with a fork. The inside stent gradually moved toward the papilla as the forceps was being rotated and was then removed through the endoscope ( $\bullet$ Video 1 ). Finally, a new inside stent (Through \&
Pass) of the same size as the previous one was placed across the stricture. The procedure was completed without complications.

Benign biliary strictures are a clinically common and serious complication, occurring in $30 \%-40 \%$ of patients undergoing LDLT [1,2]. Inside stents have long-term patency compared with conventional stents that are placed across the duodenal papilla, because of their reduced reflux of duodenal contents [3, 4]. This suggests that inside stent placement might be the first-line treatment for post-LDLT biliary strictures. Recently, threaded inside stents have become commercially available to facilitate inside stent removal; however, because the thread is made of nylon, it may slip if it is merely grabbed. Because the thread can be difficult to grasp with a device or can break, stent removal can be difficult. The method presented in this report appears safer and more reliable than merely grabbing the thread.

Endoscopy_UCTN_Code_TTT_1AR_2AZ 


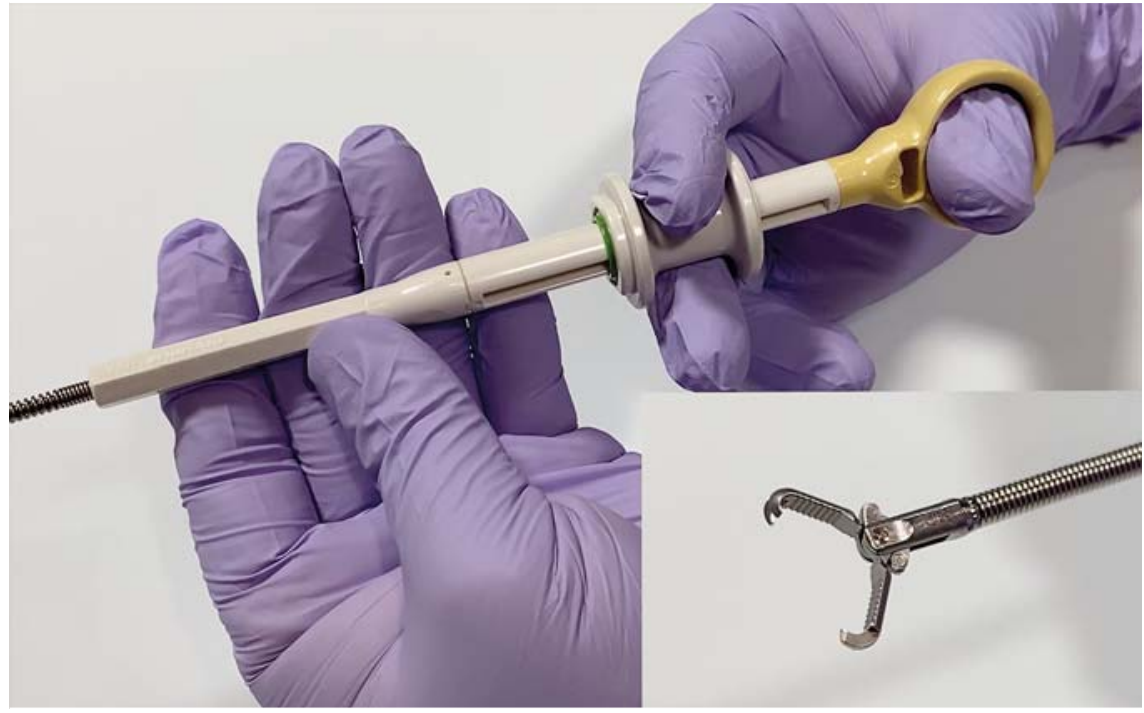

- Fig. 4 Image of the rotatable grasping forceps (FG-44NR-1) that was used to grip the thread, before rotating to roll the thread and pull the stent toward the papilla.
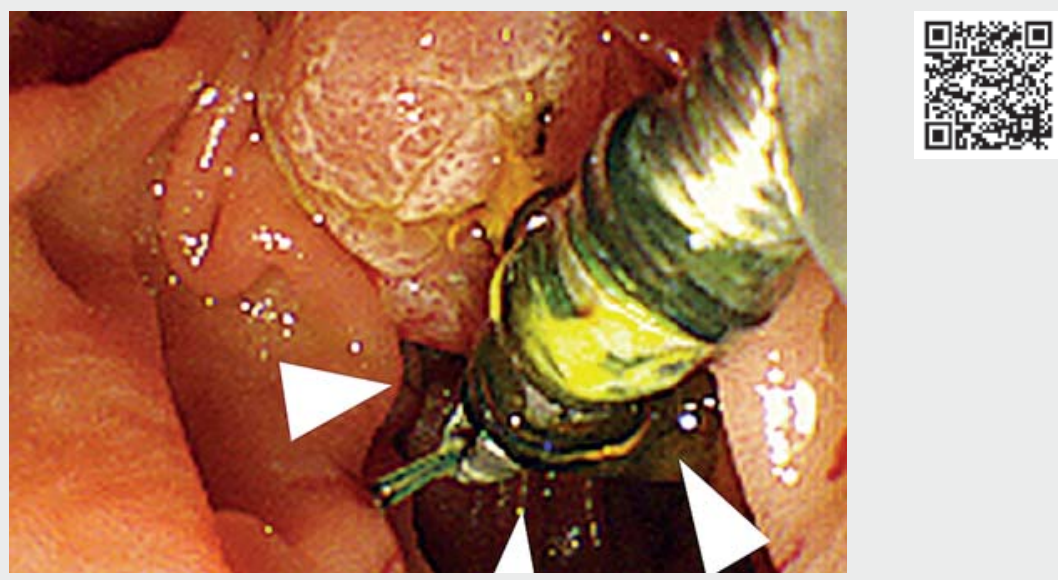

Video 1 Video showing the inside stent removal procedure. The duodenoscopy view shows the inside stent thread being tightly rolled around the forceps, pulling the stent to the papilla for removal.

\section{Competing interests}

The authors declare that they have no conflict of interest.

The authors

Masataka Yokode ${ }^{1}$, Tomoaki Matsumori Norimitsu Uza, Hirokazu Okada @ Takeshi Kuwada, Masahiro Shiokawa, Hiroshi Seno Department of Gastroenterology and Hepatology, Graduate School of Medicine, Kyoto University, Kyoto

\section{References}

[1] Zimmerman MA, Baker T, Goodrich NP et al. Development, management, and resolution of biliary complications after living and deceased donor liver transplantation: A report from the adult-to-adult living donor liver transplantation cohort study consortium. Liver Transpl 2013; 19: 259-267

[2] Chang JH, Lee IS, Choi JY et al. Biliary stricture after adult right-lobe living-donor liver transplantation with duct-to-duct anastomosis: long-term outcome and its related factors after endoscopic treatment. Gut Liver 2010; 4: 226-233

[3] Kurita A, Kodama Y, Minami R et al. Endoscopic stent placement above the intact sphincter of Oddi for biliary strictures after living donor liver transplantation. J Gastroenterol 2013; 48: 1097-1104

[4] Koizumi M, Kumagi T, Kuroda T et al. Endoscopic stent placement above the sphincter of Oddi for biliary strictures after living donor liver transplantation. BMC Gastroenterol 2020; 20: 92

\section{Bibliography}

Endoscopy 2022; 54: E496-E497

DOI 10.1055/a-1640-8829

ISSN 0013-726X

published online 8.10 .2021

(C) 2021. Thieme. All rights reserved.

Georg Thieme Verlag KG, Rüdigerstraße 14, 70469 Stuttgart, Germany

\section{CORRECTION}

A unique technique for the removal of threaded biliary inside plastic stents

Yokode M, Matsumori T, Uza N et al. A unique technique for the removal of threaded biliary inside plastic stents. Endoscopy 2021, doi:10.1055/a-16408829

In the above-mentioned article, the institution of the authors has been corrected. This was corrected in the online version on October 21, 2021. 